\title{
All-Optical Switches Based on $3 \times 3$ Multimode Interference Couplers Using Nonlinear Directional Couplers
}

\author{
Cao Dung Truong ${ }^{1}$, Trung Thanh $\mathrm{Le}^{2} \&$ Duc Han Tran ${ }^{1}$ \\ ${ }^{1}$ School of Electronics and Telecomunications, Hanoi University of Science and Technology, Hanoi, Vietnam \\ ${ }^{2}$ Facultyl of Information Technology, Hanoi University of Natural Resource and Environment, Hanoi, Vietnam \\ Correspondence: Cao Dung Truong, School of Electronics and Telecommunications, Hanoi University of \\ Science and Technolog, No1 Dai Co Viet street, Hai Ba Trung district, Hanoi, Vietnam. Tel: 84-936-354-555. \\ E-mail: dungtc.vtn1@vnpt.vn
}

Received: March 17, 2013 Accepted: April 7, 2013 Online Published: May 1, 2013

doi:10.5539/apr.v5n3p58 URL: http://dx.doi.org/10.5539/apr.v5n3p58

\begin{abstract}
Multimode interference in optical waveguide is attractive for all optical switching. In this paper, a novel $3 \times 3$ all-optical switch based on $3 \times 3$ multimode interference (MMI) structures is proposed. Nonlinear directional couplers in two arms of the structure are used as phase shifters. In this study, we use chalcogenide glass on silica for designing the device structure. The switching states of the device can be controlled by adjusting the optical control signals at the phase shifters. The transfer matrix method and beam propagation method (BPM) are used for designing and optimizing the device structure.
\end{abstract}

Keywords: all optical switch, MMI coupler, nonlinear directional coupler, phase shifter, chalcogenide glass

\section{Introduction}

Optical communication networks have evolved into the era of all optical networks. In such networks, all-optical switching elements are particularly important. In recent years, various approaches to realize all optical switches have been proposed. The MEMS switch is one of the best candidates for large order switch systems. In addition, the thin film based switch (Kruger, Joushaghani, \& Poon, 2012) (expensive for packaging and difficult to integrate with other devices), the liquid crystal based switch, the directional coupler based switch (Sugisaka, Yamamoto, Okano, \& Komori, 2008) and the MMI coupler based switch have been either commercially available or found in laboratories. In comparison with other all optical switches, the MMI based switch has the advantages of low loss, ultra-compact size, high stability, large fabrication tolerance and greater feasibility for integration (Leuthold, Besse, Hess, \& Melchior, 1997). There are many implementation methods to realize optical switching based on MMI structures (Cahill, 2005). For switching purposes, MMIs can either be placed in a Mach-Zehnder interferometer (MZI) as splitter or used as distinct region. Although, there have been some optical switches using MMI structures using thermo-optic (Al-Hetar, Mohammad, Supa'at, \& Shamsan, 2011; Liu, Mak, \& Wong, 2009) and electro-optic effects (Earnshaw \& Allsopp, 2010; Wang \& Yao, 2007). However, high speed optical communication systems require high speed optical switches. Therefore, it is particularly necessary to achieve all-optical switches.

Recently, chalcogenide $\left(\mathrm{As}_{2} \mathrm{~S}_{3}\right)$ waveguides have been proposed as a new platform for optical signal processing offering superior performance at ultrahigh bit-rates. Additionally, the high nonlinearity enables compact components with the potential for monolithic integration (Xu et al., 2008), owing to its large nonlinear coefficient $\mathrm{n}_{2}$ and low two-photon absorption (good figure of merit), the ability to tailor material properties via stoichiometry, as well as its photosensitivity. These properties allow the fabrication of photowritten gratings and waveguides.

The main aim of this paper is to propose a new structure for all-optical switching based on two $3 \times 3$ MMI couplers using nonlinear directional couplers as phase shifters. Chalcogenide glass on silica platform is used for our designs. In this work, the operating principle of MMI based switches using theoretical analysis is presented. Nonlinear directional couplers at two outermost arms in the inter-stage of two $3 \times 3$ MMI couplers play the role of phase shifters. In order to realize the phase shifters using nonlinear directional couplers, the control signal is at an arm of the nonlinear directional coupler, and the information signal is at the other arm that is also the 
outermost arm of the MZI structure. The control signal must be separated from input signals and enters the switching structure from a different single-mode access waveguide after the switching operation. The aim is to reduce the powers transferring between control waveguides and information signal waveguides. Numerical simulations are used to verify the operating principle of the proposed all-optical switch.

\section{Device Design and Analysis}

\subsection{Analytical Expression of the $3 \times 3$ MMI Coupler}

The operation of optical MMI coupler is based on the self-imaging principle (Bachmann, Besse, \& Melchior, 1994). Self-imaging is a property of a multimode waveguide by which input field is reproduced in single or multiple images at periodic intervals along propagation direction of the waveguide. MMI coupler can be characterized by the transfer matrix theory (Bachmann, Besse, \& Melchior, 1994; Soldano \& Pennings, 1995). Following this theory, the relationship between the input vector and output vector can be obtained. To achieve the required transfer matrix, the positions of the input and output ports of the MMI coupler must be set exactly.

In this study, the $3 \times 3 \mathrm{MMI}$ waveguide has a width of $\mathrm{W}_{\mathrm{MMI}}$, the access waveguides have the same width of $\mathrm{W}_{\mathrm{a}}$. The positions of three input and output ports are located at $\mathrm{x}_{\mathrm{i}}$

$$
x_{i}=\left(i+\frac{1}{2}\right) \frac{W_{e}}{3},(i=0,1,2)
$$

where $\mathrm{W}_{\mathrm{e}}$ is the effective width of the MMI coupler. It is determined by $\mathrm{W}_{\mathrm{e}}=\mathrm{W}+\frac{\lambda_{0}}{\pi}\left(\mathrm{n}_{\mathrm{r}}^{2}-\mathrm{n}_{\mathrm{c}}^{2}\right)^{-0.5}$.

Here $n_{r}$ is the refractive index of the core layer; $\lambda_{0}$ is the free space wavelength.

In the general interference mechanism, the shortest length of the MMI coupler is set by

$$
\mathrm{L}_{\mathrm{MMI}}=\mathrm{L}_{\pi}
$$

where $\mathrm{L}_{\pi}$ is the half-beat length of two lowest-order modes and can be written as

$$
\mathrm{L}_{\pi}=\frac{\pi}{\beta_{0}-\beta_{1}} \approx \frac{4 \mathrm{n}_{\mathrm{r}} \mathrm{W}_{\mathrm{e}}^{2}}{3 \lambda_{0}}
$$

The transfer matrix of the $3 \times 3$ general interference couplers (GI-MMI) is determined by (Bachmann, Besse, $\&$ Melchior, 1994)

$$
M=\frac{1}{\sqrt{3}}\left(\begin{array}{ccc}
e^{-j \frac{\pi}{3}} & e^{j \frac{2 \pi}{3}} & e^{j \frac{\pi}{3}} \\
e^{j \frac{2 \pi}{3}} & e^{j \frac{\pi}{3}} & e^{j \frac{2 \pi}{3}} \\
e^{j \frac{2 \pi}{3}} & e^{-j \frac{\pi}{3}}
\end{array}\right)
$$

\subsection{All-Optical Switch Using $3 \times 3$ MMI Structures}

The configuration of the proposed all-optical switching is shown in Figure 1 . It consists of two $3 \times 3$ general interference MMI couplers having the same size. The first MMI operates as a splitter whilst the second operates as a combiner. Here, two nonlinear directional couplers are used as two phase shifters for switching input signals from input ports $a_{i}(i=1,2,3)$ to output ports $b_{j}(j=1,2,3)$. 


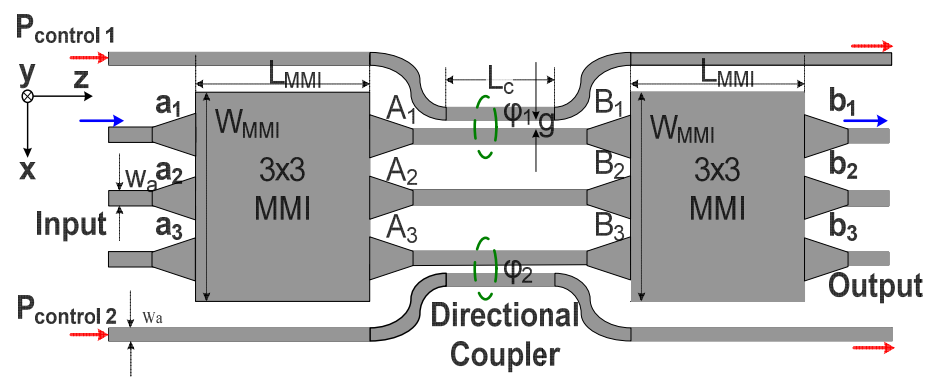

Figure. 1. An all optical switching based on $3 \times 3 \mathrm{MMI}$ couplers using directional couplers as phase shifters

Complex amplitudes and phase shifters at input and output ports of the proposed structure (see on Figure 1) can be expressed by the following matrices

$$
M_{\text {in }}=\left(\begin{array}{l}
a_{1} \\
a_{2} \\
a_{3}
\end{array}\right), M_{\text {out }}=\left(\begin{array}{l}
b_{1} \\
b_{2} \\
b_{3}
\end{array}\right) \text { and } \Phi=\left(\begin{array}{ccc}
\mathrm{e}^{j \varphi_{1}} & 0 & 0 \\
0 & 1 & 0 \\
0 & 0 & \mathrm{e}^{j \varphi_{2}}
\end{array}\right)
$$

Where $a_{1}, a_{2}, a_{3}$ and $b_{1}, b_{2}, b_{3}$ are three input ports and output ports respectively; $\varphi_{1}$ and $\varphi_{2}$ caused by directional couplers respectively are relative phase shifting angles between at two outermost arms which link MMI regions (in the condition of initial phases at ports $A_{1}, A_{2}$ and $A_{3}$ are considered the same values, respectively).

We have the following relations:

$$
\mathrm{M}_{\text {out }}=\mathrm{M} \cdot \Phi \cdot \mathrm{M} \cdot \mathrm{M}_{\text {in }}
$$

Equation (6) can be written in detail

$$
\left(\begin{array}{l}
b_{1} \\
b_{2} \\
b_{3}
\end{array}\right)=\frac{1}{3}\left(\begin{array}{ccc}
e^{j\left(\varphi_{1}-\frac{\pi}{3}\right)} & e^{j \frac{2 \pi}{3}} & e^{j\left(\varphi_{2}+\frac{\pi}{3}\right)} \\
e^{j\left(\varphi_{1}+\frac{2 \pi}{3}\right)} & e^{j \frac{\pi}{3}} & e^{j\left(\varphi_{2}+\frac{2 \pi}{3}\right)} \\
e^{j\left(\varphi_{1}+\frac{\pi}{3}\right)} & e^{j \frac{2 \pi}{3}} & e^{j\left(\varphi_{2}-\frac{\pi}{3}\right)}
\end{array}\right) \cdot\left(\begin{array}{ccc}
e^{-j \frac{\pi}{3}} & e^{j \frac{2 \pi}{3}} & e^{j \frac{\pi}{3}} \\
e^{j \frac{2 \pi}{3}} & e^{j \frac{\pi}{3}} & e^{j \frac{2 \pi}{3}} \\
e^{j \frac{\pi}{3}} & e^{j \frac{2 \pi}{3}} & e^{-j \frac{\pi}{3}}
\end{array}\right) \cdot\left(\begin{array}{l}
a_{1} \\
a_{2} \\
a_{3}
\end{array}\right)
$$

Table 1. Phase shift states for operation of the $3 \times 3$ optical switches

\begin{tabular}{cccc}
\hline Input & $\varphi_{1}$ & $\varphi_{2}$ & Output \\
\hline $\mathrm{a}_{1}$ & 0 & $2 \pi / 3$ & $\mathrm{~b}_{1}$ \\
$\mathrm{a}_{1}$ & $2 \pi / 3$ & 0 & $\mathrm{~b}_{2}$ \\
$\mathrm{a}_{1}$ & $-2 \pi / 3$ & $-2 \pi / 3$ & $\mathrm{~b}_{3}$ \\
$\mathrm{a}_{2}$ & $2 \pi / 3$ & 0 & $\mathrm{~b}_{1}$ \\
$\mathrm{a}_{2}$ & $-2 \pi / 3$ & $-2 \pi / 3$ & $\mathrm{~b}_{2}$ \\
$\mathrm{a}_{2}$ & 0 & $2 \pi / 3$ & $\mathrm{~b}_{3}$ \\
$\mathrm{a}_{3}$ & $-2 \pi / 3$ & $-2 \pi / 3$ & $\mathrm{~b}_{1}$ \\
$\mathrm{a}_{3}$ & 0 & $2 \pi / 3$ & $\mathrm{~b}_{2}$ \\
$\mathrm{a}_{3}$ & $2 \pi / 3$ & 0 & $\mathrm{~b}_{3}$ \\
\hline
\end{tabular}

Now we calculate the phase shifters to control input signals to any output ports. Assuming that output is switched to port $b_{1}$ (that means $b_{1}=1, b_{2}=b_{3}=0$ ) from port $a_{1}\left(a_{1}=1, a_{2}=a_{3}=0\right)$. Expanding and solving the Equations (7) we can easily obtain the roots $\varphi_{1}=0, \varphi_{2}=2 \pi / 3$. In the same ways, we also find out matched phase shifts for all switching operation states. In summary, in the condition of phase shifts are caused by the first MMI, 
accumulation phases at shifters required to control the input signal to any output ports can be expressed in Table 1.

As mentioned above, the structure of an all optical switching requires two nonlinear directional couplers (Danaie \& Kaatuzian, 2011) as phase shifters at two outermost arms of optical device as shown in Figure 1. Originally, the nonlinear directional coupler includes two waveguides that have small distance and full coupling takes place between them in one coupling length, provided that one or both of them have non-linear behavior. This non-linear behavior can be guaranteed with high intensity control field which changes the nonlinear refractive index. When the distance of two nonlinear directional couplers is very small and mode field amplitudes vary slowly in the $\mathrm{z}$ - propagation direction, the interaction of electrical fields in nonlinear directional couplers complies with coupled mode equations

$$
\begin{aligned}
& -i \frac{d A}{d z}=\kappa B+\gamma_{1}\left(|A|^{2}+2|B|^{2}\right) A \\
& -i \frac{d B}{d z}=\kappa A+\gamma_{2}\left(|B|^{2}+2|A|^{2}\right) B
\end{aligned}
$$

Where $\kappa$ is the linear coupling coefficient, it is determined by $\kappa=\frac{\pi}{2 L_{c}}, L_{c}$ is coupling length, A and B are field amplitudes of the control and signal waveguides of the directional coupler and $\gamma_{1}, \gamma_{2}$ are nonlinear coefficients describing the self-phase modulation (SPM) and cross-phase modulation (XPM) effects. Nonlinear coefficient is determined as follow

$$
\gamma=\frac{2 \pi \mathrm{n}_{2}}{\lambda_{0} \mathrm{~A}_{\mathrm{eff}}}
$$

$\lambda_{0}$ is wavelength in the vacuum, $\mathrm{n}_{2}$ is nonlinear refractive index of the waveguide, $\mathrm{A}_{\text {eff }}$ is the effective modal cross-section area. Under the effect of self-phase modulation in the nonlinear directional coupler, the phase in directional coupler can be changed proportional to the intensity of input of electrical fields of waveguides.

The nonlinear phase shifts in the directional-coupler waveguides that are caused by cross phase modulation (XPM) effect can be defined as

$$
\varphi=\frac{2 \pi \mathrm{n}_{2} \mathrm{~L}_{\mathrm{c}}\left(\mathrm{I}_{0}+2 \mathrm{I}_{\mathrm{c}}\right)}{\lambda_{0}}
$$

Where: $I_{0}, I_{c}$ are the intensities of signal and control waveguides respectively. In the phase matched case when the input wavelength and the refractive index of two waveguides are identical, maximum coupling will take place.

\subsection{Design and Optimization}

In this study, we use the chalcogenide glass $\mathrm{As}_{2} \mathrm{~S}_{3}$ for designing the whole device. The material used in core layer of the proposed optical switching structure is chalcogenide glass $\mathrm{As}_{2} \mathrm{~S}_{3}$ with refractive index $\mathrm{n}_{\mathrm{r}}=2.45$ and the silica material $\mathrm{SiO}_{2}$ used in cladding layer has refractive index $\mathrm{n}_{\mathrm{c}}=1.46 . \mathrm{As}_{2} \mathrm{~S}_{3}$ (arsenic trisulfide) is a direct band-gap, amorphous semiconductor. By using a highly controlled deposition process, a photo-polymerizable film of $\mathrm{As}_{2} \mathrm{~S}_{3}$ can be deposited on standard silica glass substrates. Chalcogenide $\left(\mathrm{As}_{2} \mathrm{~S}_{3}\right)$ is chosen due to its advantages. For example, it is attractive for high rate photonics integrated circuits (Pelusi, Luan, Madden, Choi, Bulla, Member, \& Eggleton, 2010; Ta et al., 2007), especially attractive for all optical switches in recent years because of the fast response time associated with the near-instantaneous third order nonlinearity allows flexible ultrafast signal processing. In addition, the chalcogenide glass supports the operation of wavelengths range in the windows $1.55 \mu \mathrm{m}$; and $\mathrm{As}_{2} \mathrm{~S}_{3}$ material has a high refractive index contrast to allow for a high confinement of light (Shi, Anand, \& He, 2009) also ultra-compact size. Therefore, it is useful and important for large scale integrated circuits. The other advantage of the chalcogenide glass is that it has a high nonlinear coefficient $\mathrm{n}_{2}$ about $2.92 \times 10^{-6} \mu \mathrm{m}^{2} / \mathrm{W}$. From Equation (11), we can see that phase angle in the phase shifter of the structure increases proportionally in the nonlinear coefficient and the control field intensity, so if nonlinear coefficient is high then control field intensity is low when the phase angle is constant. This is better for operation of the proposed switch because a very high intensity of the control beam will overwhelm the signal. Moreover, since the control beam intensity is much higher than the signal beam one, the nonlinear directional coupler needs an extreme high isolation; so that it is difficult to design and optimize the proposed structure. Silicon dioxide $\mathrm{SiO}_{2}$ is used in cladding layer because of high refractive index difference between core and cladding layers that allows 
for a high confinement of light and also supports a larger mode numbers in MMI region. In addition, both $\mathrm{As}_{2} \mathrm{~S}_{3}$ and $\mathrm{SiO}_{2}$ materials are available and cheap also they can implement in the practical fabrication. Recently, these materials are very attractive for ultrahigh bit-rate signal processing applications (Finsterbusch, Baker, Ta, Eggleton, Choi, \& Madden, 2006; Luan, Pelusi, Lamont, Choi, Madden, Luther-Davies, \& Eggleton, 2009).

The device used in our designs is shown on Figure 1. Here, we use the TE (Transverse Electric) polarization and operating wavelength 1550-nm for analyses and simulations. If the uniformity of the time harmonic of TE-polarized waves can be assumed along the $\mathrm{x}$ direction of Figure 1, the simulation can be done assuming it as a 2D structure (Bahrami, Mohammadnejad, \& Rostami, 2011; Tseng, Fuentes-Hernandez, Owens, \& Kippelen, 2007). In order to reduce time consuming but still have accurate results. a $3 \mathrm{D}$ device structure is converted to a 2D structure using the effective index method (EIM) first, then the 2D-BPM method is used for simulations (Tseng, Fuentes-Hernandez, Owens, \& Kippelen, 2007).

The design parameters of the proposed structure are chosen as follows: the width of each $3 \times 3$ MMI coupler $\mathrm{W}_{\text {MMI }}$ is $24 \mu \mathrm{m}$, the width of access waveguides $\mathrm{W}_{\mathrm{a}}$ is $4 \mu \mathrm{m}$ in order for single mode condition can be obtained, the length of the multimode region $\mathrm{L}_{\mathrm{MMI}}$ is set as $\mathrm{L}_{\pi}$ for the general interference mechanism and it can be calculated by the mode propagation analysis (MPA) method is $1259.8 \mu \mathrm{m}$.

Parameters of the control waveguides are designed as follows: the width is set as $\mathrm{W}_{\mathrm{a}}$; at the beginning, a straight waveguide has the length of $2059.15 \mu \mathrm{m}$ calculated by using the BPM. Next, it is connected to a sine waveguide which has the length of $1000 \mu \mathrm{m}$ in z propagation direction and the distance of $9 \mu \mathrm{m}$ in $\mathrm{x}$-direction. Then it is concatenated to another straight waveguide. By using the BPM, the length of the straight waveguide of the nonlinear directional couplers $\mathrm{L}_{\mathrm{c}}$ is chosen to be $360 \mu \mathrm{m}$ to satisfy the eliminating condition of the cross transfer power between control and structure waveguides. Gap g between this straight waveguide and the outermost arm is small (Figure 1) to enable mode coupling. Finally, a sine waveguide and a straight waveguide are in turn connected (as shown on Figure 1). We choose the sine waveguide for two purposes: first, the sine waveguides are used to connect the straight waveguides together in which it puts a waveguide near outermost arms which link between MMI regions in order to make a full coupling and a phase shift between nonlinear directional waveguides and the second aim is that light beam power can be conserved when propagated through it. Both control beams and input signal beams have the same wavelength, amplitude and polarization state in all of switching states.

Now we optimize the whole device structure. Firstly, the length $\mathrm{L}_{\mathrm{MMI}}$ is optimized by the 2D-BPM method to find the optimal value by changing the values of the length around $\mathrm{L}_{\pi}$. Finally, we find out the optimal value as $1260 \mu \mathrm{m}$. The optimal gap g between two parallel waveguides of the directional couplers used as phase shifters can be found by using the BPM. The simulations are shown in Figure 2. We need to find the optimal value $g$ to minimize the cross transferring power between outermost arms and the control waveguides and split the total power entering into one input port equally at mode coupling positions in case of the control waveguide is on and access waveguides are on one by one into 3 arms $A_{1} B_{1}, A_{2} B_{2}, A_{3} B_{3}$ as $P_{A 1 B 1}, P_{A 2 B 2}, P_{A 3 B}$, respectively. This can be done by introducing power into ports $a_{1}, a_{2}$ and $a_{3}$ and use 2D-BPM method. Due to the symmetry of the proposed structure, we only need to consider the power inserted into control waveguide 1 . By changing the value of g gradually from $0.09 \mu \mathrm{m}$ to $0.11 \mu \mathrm{m}$ and monitoring and normalizing the power $\mathrm{P}_{\mathrm{A} 1 \mathrm{~B} 1}$ as well as $\mathrm{P}_{\text {controll }}$, we choose the optimal value of $\mathrm{g}$ as $0.1 \mu \mathrm{m}$ according to Figure 2.

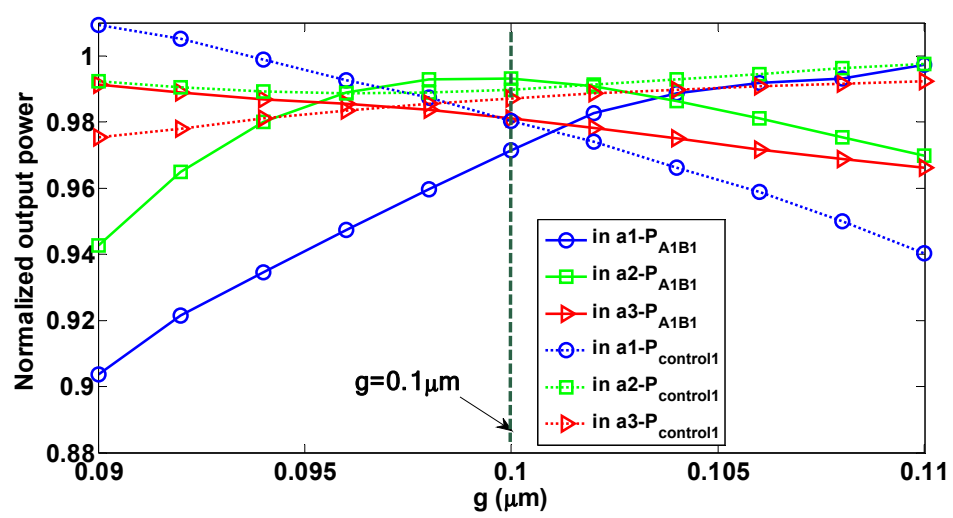

Figure 2. 2D BPM simulation results for the optimal values of the gap g between control and structure waveguide 
To optimize the operation of the MMI regions in the role of the splitter and combiner as well as minimize the insertion loss and crosstalk effect, linear taper waveguides are used to connect between MMI regions and access waveguides. In our design, linear tapers have the length $l_{a}=150 \mu \mathrm{m}$ and the widths from $3 \mu \mathrm{m}$ to $5 \mu \mathrm{m}$ are calculated and optimized by BPM simulations.

As mentioned before in results are shown on the Table 1 , when the input field enters the switch from the input $\mathrm{a}_{1}$ port, if the phase shift in the first linking arm is zero and the second linking arm is $2 \pi / 3$, it will switch to output $b_{1}$ port.

Table 2. Power intensity states for operation of the $3 \times 3$ optical switch

\begin{tabular}{cccc}
\hline Input & Output & $\begin{array}{c}\text { Optimal } \\
\mathrm{I}_{1} \\
\left(\mathrm{GW} / \mathrm{cm}^{2}\right)\end{array}$ & $\begin{array}{c}\text { Optimal } \\
\mathrm{I}_{2} \\
\left(\mathrm{GW} / \mathrm{cm}^{2}\right)\end{array}$ \\
\hline $\mathrm{a}_{1}$ & $\mathrm{~b}_{1}$ & 450 & 277 \\
$\mathrm{a}_{1}$ & $\mathrm{~b}_{2}$ & 274.2 & 450.75 \\
$\mathrm{a}_{1}$ & $\mathrm{~b}_{3}$ & 330.12 & 332.62 \\
$\mathrm{a}_{2}$ & $\mathrm{~b}_{1}$ & 279 & 448.5 \\
$\mathrm{a}_{2}$ & $\mathrm{~b}_{2}$ & 327.8 & 327.8 \\
$\mathrm{a}_{2}$ & $\mathrm{~b}_{3}$ & 448.5 & 279 \\
$\mathrm{a}_{3}$ & $\mathrm{~b}_{1}$ & 332.62 & 330.12 \\
$\mathrm{a}_{3}$ & $\mathrm{~b}_{2}$ & 450.75 & 274.2 \\
$\mathrm{a}_{3}$ & $\mathrm{~b}_{3}$ & 277 & 450 \\
\hline
\end{tabular}

For switching from an input to an output of the structure, we implement numerical simulation by 2D-BPM method to find optimal values of field intensities of control waveguides. The simulation has to satisfy two requirements: the first, we find the values of field intensities of control waveguides to produce exactly matched phase shifts for switching operations; then those values must be optimized so that the transfer power between signal waveguides and control waveguides is minimal.

We assume that the normalized input power in optical switching device is set as 1 normalized unit; input field intensity $\mathrm{I}_{0}$ equals $1 \mathrm{GW} / \mathrm{cm}^{2}$. This value is chosen because it can generate the largest nonlinear phase shift (Ruan, Luther-Davies, Li, Rode, Kolev, \& Madden, 2005). To reach the switching state from port $\mathrm{a}_{1}$ to port $\mathrm{b}_{1}$, firstly we find the intensity $I_{1}$, which is introduced into control waveguide 1 (also see Figure 1), by varying the intensity slowly. The appropriate result is about $448 \mathrm{GW} / \mathrm{cm}^{2}$ making phase shift 0 in comparison with the center access waveguide. Secondly, we can also change the value of the intensity $\mathrm{I}_{2}$, which is introduced into control waveguide 2. The appropriate result is about $279.6 \mathrm{GW} / \mathrm{cm}^{2}$ making phase shift $2 \pi / 3$ in comparison with the center access waveguide. Finally, if we use these results to reproduce the simulation and adjust their values very slowly around them again, we obtain the optimal values $\mathrm{I}_{1}=450 \mathrm{GW} / \mathrm{cm}^{2}$ and $\mathrm{I}_{2}=277 \mathrm{GW} / \mathrm{cm}^{2}$, respectively. The reason for this is due to the loss when the light travels in the MMI region and also because the length of MMI region is too long to be operated as a splitter or a combiner accurately. Table 2 lists optimal field intensities and states of control waveguides used in two control waveguides.

\section{Simulation and Discussion}

Figure 3 shows simulation results by 2 D BPM for all of switching states in the $3 \times 3$ all optical switches corresponding to all values of parameters listed on the Table 2 . With respect to visual geometry, the results are good for operation states of the switch. Due to symmetric nature of the proposed structure, the role of input ports $a_{1}$ and $a_{3}$ are equivalent. Without loss of generality, we only need to consider the simulation results of $a_{1}$ and $a_{2}$ ports of the structure.

Results reveal high output power intensity, which ensures the high performance of the switching structure. Subsequently, a high-level switch should have the suitable insertion loss, extinction ratio, crosstalk, and good tolerance independency against the wavelength and fabrication. Thus indicating the listed parameters is important in manufacturing an optical switch. 
Table 3. Insertion Loss, extinction ratio and crosstalk of the proposed structure

\begin{tabular}{ccccc}
\hline Input & Output & $\begin{array}{c}\text { Insertion } \\
\text { Loss }(\mathrm{dB})\end{array}$ & $\begin{array}{c}\text { Extinction } \\
\text { Ratio }(\mathrm{dB})\end{array}$ & $\begin{array}{c}\text { Crosstalk } \\
(\mathrm{dB})\end{array}$ \\
\hline a1 & b1 & -0.19 & 31.94 & 28.65 \\
a1 & b2 & -0.3 & 30.73 & 28.83 \\
a1 & b3 & -0.15 & 33.33 & 28.69 \\
a2 & b1 & -0.17 & 33.16 & 28.67 \\
a2 & b2 & -0.1 & 29.37 & 29.03 \\
a2 & b3 & -0.17 & 33.16 & 28.67 \\
a3 & b1 & -0.15 & 32.33 & 28.69 \\
a3 & b2 & -0.3 & 30.73 & 28.83 \\
a3 & b3 & -0.19 & 31.94 & 28.65 \\
\hline
\end{tabular}
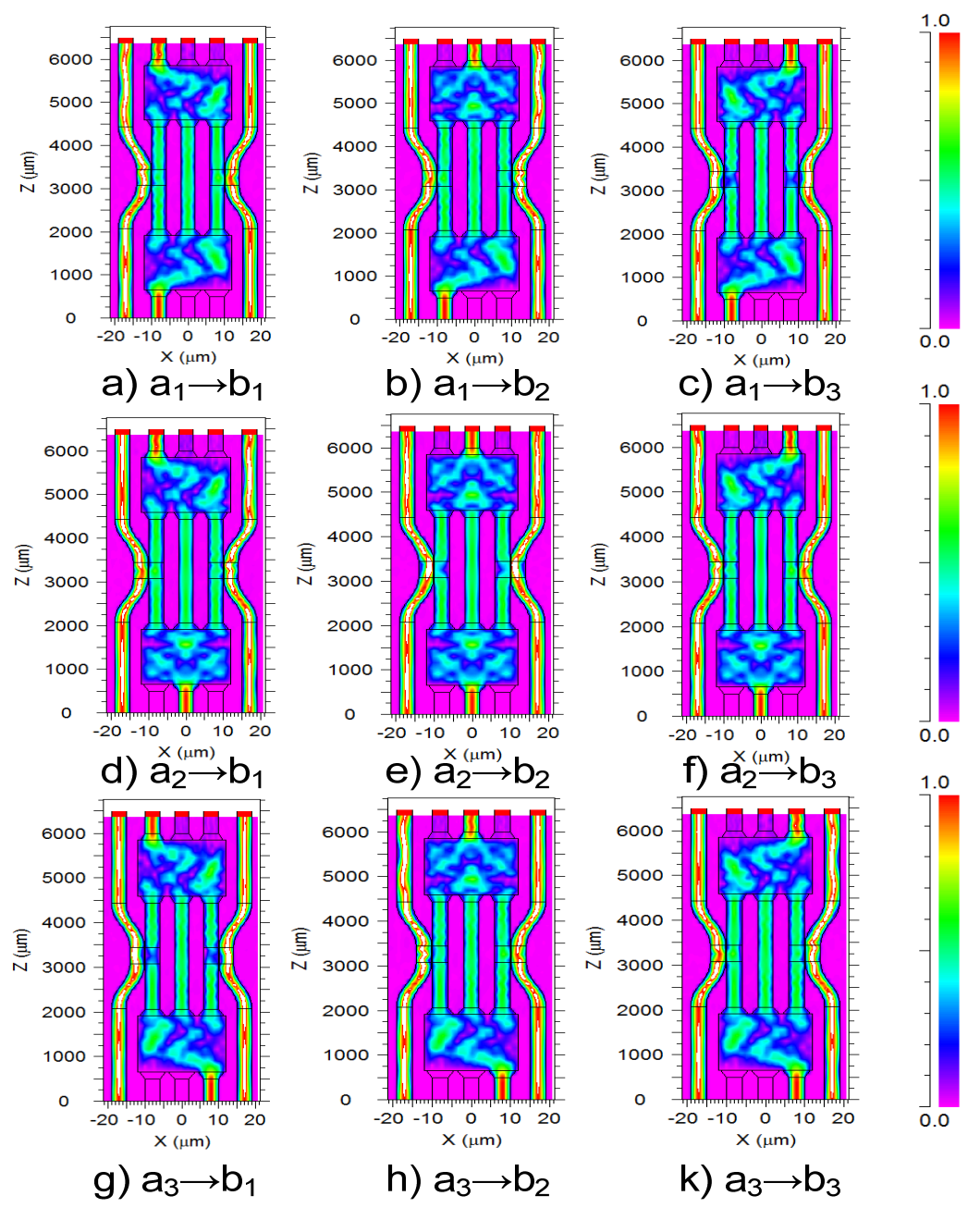

h) $a_{3} \rightarrow b_{2}$

k) $a_{3} \rightarrow b_{3}$

Figure 3. Simulation results implemented by BPM method for all switching states of the $3 \times 3$ all optical switches 
The calculation formulas for the insertion loss (I. L.) and extinction ratio (Ex. R.) as follows (Bahrami, Mohammadnejad, \& Rostami, 2011).

$$
\begin{array}{r}
\text { I.L. }(\mathrm{dB})=10 \log _{10}\left(\frac{\mathrm{P}_{\text {out }}}{\mathrm{P}_{\text {in }}}\right) \\
\text { Ex.R. }(\mathrm{dB})=10 \log _{10}\left(\frac{\mathrm{P}_{\text {high }}}{\mathrm{P}_{\text {low }}}\right)
\end{array}
$$

Where $\mathrm{P}_{\text {out }}$ and $\mathrm{P}_{\text {in }}$ are the output and input power of the switch in operation state, $\mathrm{P}_{\text {high }}$ and $\mathrm{P}_{\text {low }}$ are output power levels in $\mathrm{ON}$ and $\mathrm{OFF}$ states of input ports respectively. Crosstalk is the ratio of the power at a specific output from the desired input to the power from all other inputs (Papadimitriou, Papazoglou, \& Pomportsis, 2003). The calculated insertion loss and extinction ratios of the switch are shown in Table 3.

Result simulation presented in Figure 3 and Table 3 prove that all of the important parameters of the proposed optical switch are suitable for all optical switching. Refractive index of $\mathrm{As}_{2} \mathrm{~S}_{3}$ in this design is calculated by Sellmeier's Equation (Chaudhari, Suzuki, \& Ohishi, 2008). Results show that when the wavelength varies from $1545 \mathrm{~nm}$ to $1555 \mathrm{~nm}$, the refractive index of As2S3 varies from 2.4496 to 2.4503. This variation is very small so we can be neglected. Therefore, in all of simulation results, we consider refractive index of chalcogenide glass as a constant.

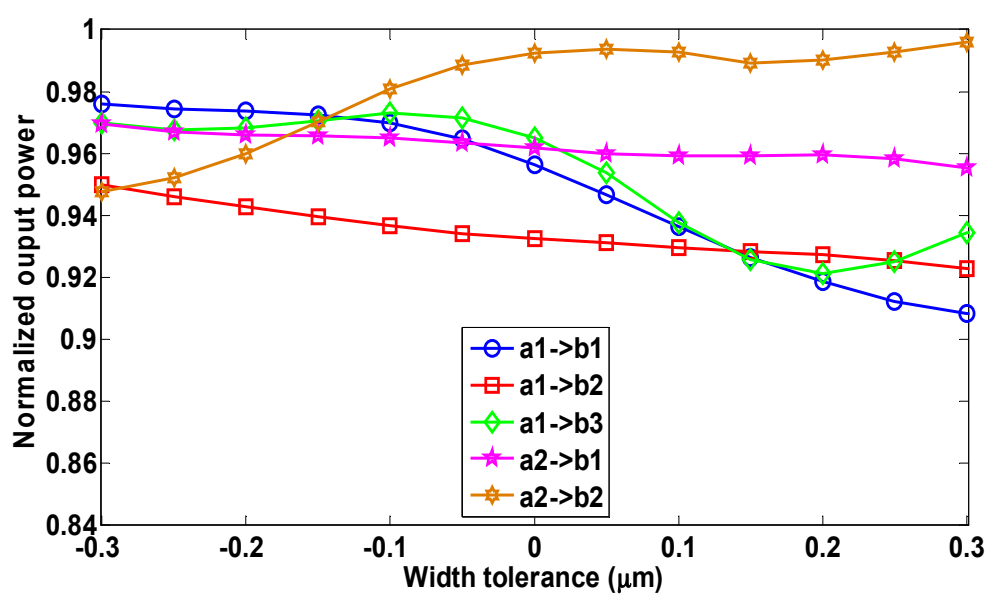

a)

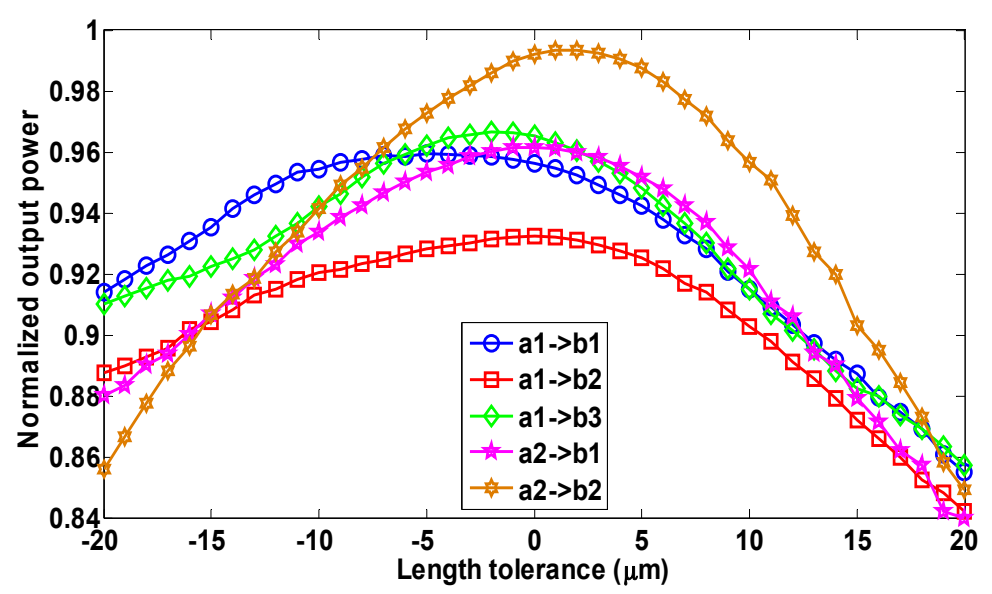

b)

Figure 4. Normalized output power on the variation of the width and length of MMI regions in operation states of the proposedswitch: a) the variation of the width and b) the variation of the length 


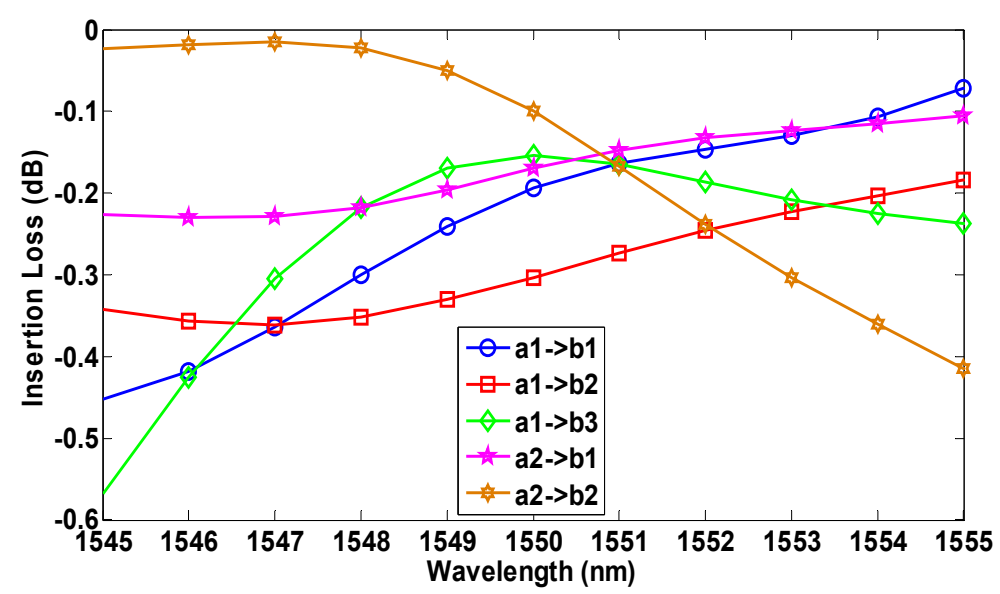

Figure 5. Dependency on wavelength of the insertion loss in operation states of proposed structure

As shown in Figure 4, the length and the width dependency of the MMI sections in proposed structure are simulated by the BPM in all of operation states of the switch. The output power is normalized according to the input power. Results denoted the variation of the output power in a quite large range of the width and the length of MMI regions. The variation of insertion loss is less than $0.75 \mathrm{~dB}$ is the tolerance of the width and the length are less than $0.3 \mu \mathrm{m}$ and $20 \mu \mathrm{m}$ respectively. Hence, the fabrication tolerance of proposed design is very large.

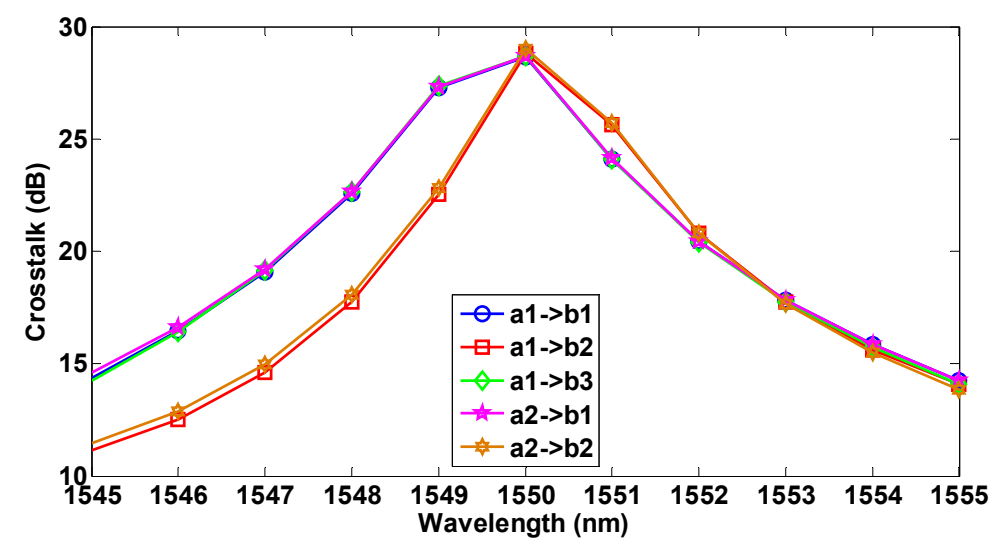

a)

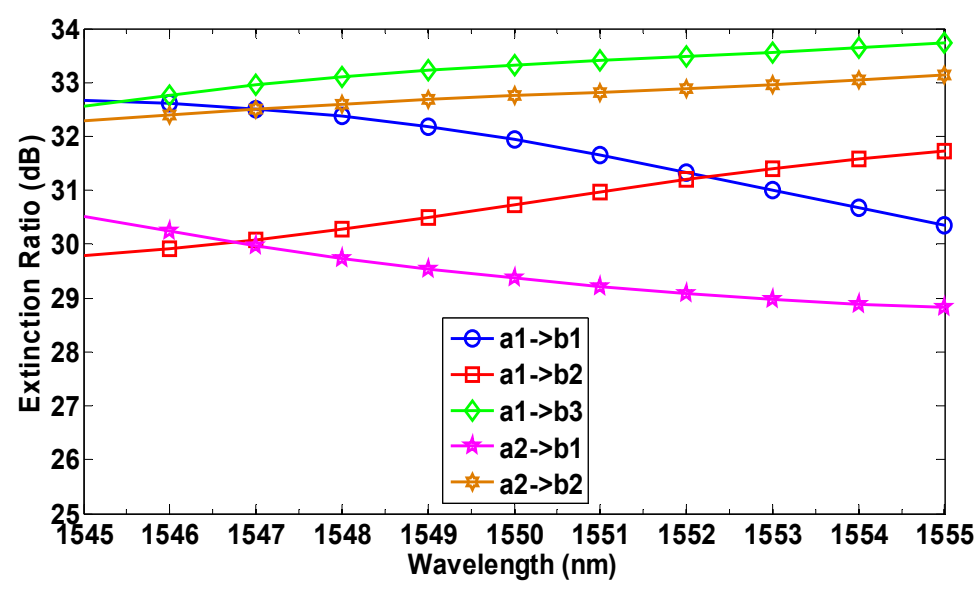

b)

Figure 6. Simulation results by 2D BPM about wavelength dependency of the proposed structure:

a) Crosstalk and b) Extinction Ratio 
Figure 5 shows the wavelength dependence of the insertion loss of the proposed all optical switch. An important parameter in operation of all optical switches is wavelength sensitivity. In this design, wavelength sensitivity is defined as the smallest change of wavelength range around the operation wavelength to ensure a variation level in output power. If we choose the variation of the output power as $0.6 \mathrm{~dB}$, the data from simulation results shows that wavelength sensitivity in all cases is $10 \mathrm{~nm}$.

Figure 6 shows the dependence of the crosstalk and extinction ratio of the switch on wavelength. The results show that in the bandwidth $10 \mathrm{~nm}$ around the center wavelength $1550 \mathrm{~nm}$, the variation of crosstalk is about 11 $\mathrm{dB}$ to $29.24 \mathrm{~dB}$ whilst the variation of extinction ratio is about $29 \mathrm{~dB}$ to $34 \mathrm{~dB}$ respectively. These values are good for applications in optical communication networks.

Clearly, the proposed switch has an ability to switch none blocking from any input ports to any output ports. In comparison with an existing $3 \times 3$ optical switch using a $3 \times 3$ fiber coupler, we can see that the $3 \times 3$ fiber coupler cannot switch none blocking between input and output ports despite having phase shift in each input port. This is explained by coupling modes into fiber couplers. The light waves in the fiber ports, as well as the structure themselves, are mirror-symmetric in one plane. Thus light in the widest fiber excites itself at the fundamental mode in the waist. Light in the intermediate fiber excites one of the second modes, and light in the narrowest fiber excites one of the third modes. In both cases, the mode is excited is the one that is symmetric about the plane of fibers. For symmetry reasons, no flexural wave can couple light between the first and third modes, so light cannot be routed directly between the widest and narrowest fibers. Hence, the single $3 \times 3$ fiber coupler is restricted set of allowed states for switching operation (Culverhouse, Birks, Farwell, \& Russell, 1997).

In comparison with the existing approach structure in the literature which used the $3 \times 3 \mathrm{MZI}$ structure and electro-optic effect (Syuhaimi, Rahman, Shaktur, \& Mohammad, 2010), our proposed structure has a better insertion loss. In addition, our proposed switch is an all-optical switch that can be useful for all-optical networks and other all-optical signal processing applications.

Moreover, in comparison with the existing MEMS technology, based on simulation results presented in Table 3, the proposed structure has the same insertion loss and crosstalk but it has some further advantages of impact size, reliability and suitability for large integrated circuits. Disadvantages of the proposed structure are polarization dependence, phase shifters driving and its manufacturing price is more expensive than MEMS switches' one (Papadimitriou, Papazoglou, \& Pomportsis, 2003).

\section{Conclusion}

A novel all-optical MMI switch has been presented in this paper. The non-linear directional couplers are utilized to realize passive phase shifters. The proposed structure are analyzed and designed by using the transfer matrix method and the beam propagation method. The optical control fields are used to make phase shifters for the operation of the all optical switch. The simulation results show that the switching operation is done carefully, the fabrication tolerance is relatively large. The proposed structure can be used to construct multi-port optical switches, such as $\mathrm{N} \times \mathrm{M}$ optical switches (where $\mathrm{N}$ and $\mathrm{M}$ are multiple of 3 ). The quality of performances of the proposed optical switch is good hence this structure can be useful for applications in optical communication networks.

\section{References}

Al-Hetar, A. M., Mohammad, A. B., Supa'at, A. S. M., \& Shamsan, Z. A. (2011). MMI-MZI Polymer Thermo-Optic Switch With a High Refractive Index Contrast. Journal of Lightwave Technology, 29(2), 171-178. http://dx.doi.org/10.1109/JLT.2010.2098473

Bachmann, M., Besse, P. A., \& Melchior, H. (1994). General self-imaging properties in $\mathrm{N} \times \mathrm{N}$ multimode interference couplers including phase relations. Applied Optics, 33(18), 3905-3911. http://dx.doi.org/10.1364/AO.33.003905

Bahrami, A., Mohammadnejad, S., \& Rostami, A. (2011). All-Optical Multi-Mode Interference Switch Using Non-Linear Directional Coupler as a Passive Phase Shifter. Fiber and Integrated Optics, 30(3), 139-150. http://dx.doi.org/10.1080/01468030.2011.576379

Cahill, L. (2005). Optical Switching Using Cascaded Generalised Mach-Zehnder Switches. TENCON 2005-2005 IEEE Region 10 Conference, 1-5, Nov. 2005. http://dx.doi.org/10.1109/TENCON.2005.300845

Chaudhari, C., Suzuki, T., \& Ohishi, Y. (2008). Chromatic Dispersions in Highly Nonlinear Glass Nanofibers. Proc. of SPIE.Photonic Fiber and Crystal Devices: Advances in Materials and Innovations in Device Applications, 7056, 1-8, Aug. 2008. http://dx.doi.org/10.1117/12.795811 
Culverhouse, D. O., Birks, T. A., Farwell, S. G., \& Russell, P. S. J. (1997). $3 \times 3$ All-Fiber Routing Switch. IEEE Photonics Technology Letters, 9(3), 333-335. http://dx.doi.org/10.1109/68.556064

Danaie, M., \& Kaatuzian, H. (2011). Improvement of power coupling in a nonlinear photonic crystal directional coupler switch. Photonics and Nanostructures Fundamentals and Applications, 9(1), 70-81. http://dx.doi.org/10.1016/j.photonics.2010.10.002

Earnshaw, M., \& Allsopp, D. (2010). Semiconductor space switches based on multimode interference couplers. Journal of Lightwave Technology, 20(4), 643-650. http://dx.doi.org/10.1109/50.996585

Finsterbusch, K., Baker, N., Ta, V. G., Eggleton, B. J., Choi, D., \& Madden, S. (2006). Long-period gratings in

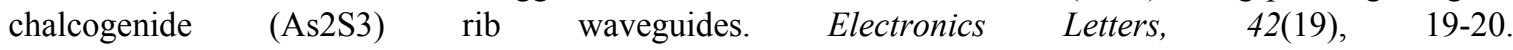
http://dx.doi.org/10.1049/el:20062257

Kruger, B. A., Joushaghani, A., \& Poon, J. K. S. (2012). Design of electrically driven hybrid vanadium dioxide (VO2) plasmonic switches. Optics Express, 20(21), 23598. http://dx.doi.org/10.1364/OE.20.023598

Leuthold, J., Besse, P. A., Hess, R., \& Melchior, H. (1997). Wide Optical Bandwidths and High Design Tolerances of Multimode-Interference Converter-Combiners Comparison with Mode-Analysis. Proc. of European Conferenc on Integrated Optics, No. 1, 154-157.

Liu, W. C., Mak, C. L., \& Wong, K. H. (2009). Thermo-optic properties of epitaxial as optical modulator. Optics Express, 17(16), 13677-13684. http://dx.doi.org/10.1364/OE.17.013677

Luan, F., Pelusi, M. D., Lamont, M. R. E., Choi, D.-Y., Madden, S., Luther-Davies, B., \& Eggleton, B. J. (2009). Dispersion engineered As2S3 planar waveguides for broadband four-wave mixing based wavelength conversion of $40 \mathrm{~Gb} / \mathrm{s}$ signals. Optics Express, 17(5), 3514-20. http://dx.doi.org/10.1364/OE.17.003514

Papadimitriou, G. I., Papazoglou, C., \& Pomportsis, A. S. (2003). Optical Switching : Switch Fabrics, Techniques, and Architectures. Journal of Lightwave Technology, 21(2), 384-405. http://dx.doi.org/10.1109/JLT.2003.808766

Pelusi, M. D., Luan, F., Madden, S., Choi, D., Bulla, D. A., Member, S., \& Eggleton, B. J. (2010). Wavelength Conversion of High-Speed Phase and Intensity Modulated Signals Using a Highly. IEEE Photonics Technology Letters, 22(1), 2009-2011. http://dx.doi.org/10.1109/LPT.2009.2035094

Ruan, Y., Luther-Davies, B., Li, W., Rode, A., Kolev, V., \& Madden, S. (2005). Large phase shifts in As2S3 waveguides for all-optical processing devices. Optics Letters, 30(19), 2605-7. http://dx.doi.org/10.1364/OL.30.002605

Shi, Y., Anand, S., \& He, S. (2009). Design of a Polarization Insensitive Triplexer Using Directional Couplers Based on Submicron Silicon Rib Waveguides. Journal of Lightwave Technology, 27(11), 1443-1447. http://dx.doi.org/10.1109/JLT.2008.2010465

Soldano, L., \& Pennings, E. C. M. (1995). Optical Multi-Mode Interference Devices Based on Self-Imaging: Principles and Applications. Journal of Lightwave Technology, 13(4), 615-627, 1995. http://dx.doi.org/10.1109/50.372474

Sugisaka, J., Yamamoto, N., Okano, M., \& Komori, K. (2008). Demonstration of a photonic crystal directional coupler switch with ultra short switching length. Conference on Lasers and Electro-Optics, May 4, 2008. http://dx.doi.org/10.1109/CLEO.2008.4551447

Syuhaimi, M., Rahman, A., Shaktur, K. M., \& Mohammad, R. (2010). Analytical And Simulation Of New Electro-Optic $3 \times 3$ Switch Using Ti : LiNbO3 As a Wave Guide Medium. International Conference on Photonics (ICP), 4-8. http://dx.doi.org/10.1109/ICP.2010.5604426

Ta, V. G., Baker, N. J., Fu, L., Finsterbusch, K., Lamont, M. R. E., Moss, D. J., ... Luther-davies, B. (2007). Ultrafast all-optical chalcogenide glass photonic circuits. Optics Express, 15(15), 5860-5865. http://dx.doi.org/10.1364/OE.15.009205

Tseng, S.-Y., Fuentes-Hernandez, C., Owens, D., \& Kippelen, B. (2007). Variable splitting ratio $2 \times 2$ MMI couplers using multimode waveguide holograms. Optics Express, 15(14), 9015-21. http://dx.doi.org/10.1364/OE.15.009015

Wang, Q., \& Yao, J. (2007). A high speed $2 \times 2$ electro-optic switch using a polarization modulator. Optics Express, 15(25), 16500-5, Dec. 2007. http://dx.doi.org/10.1364/OE.15.016500 
Xu, J., Galili, M., Mulvad, H. C. H., Oxenløwe, L. K., Clausen, A. T., Jeppesen, P., ... Eggleton, B. J. (2008). Error-free $640 \mathrm{Gbit} / \mathrm{s}$ demultiplexing using a chalcogenide planar waveguide chip. Opto-Electronics and Communications Conference, and Australian Conference on Optical Fibre Technology, OECC/ACOFT, 2, pp. 3-4, 2008.http://dx.doi.org/10.1109/OECCACOFT.2008.4610314 